\title{
AN IMPROVED METHOD FOR DUAL TRIGONOMETRICAL SERIES
}

\author{
by C. J. TRANTER
}

(Received 12 February, 1963)

1. In a previous paper in this journal [1], I gave formulae for determining the coefficients in certain dual trigonometrical series. The derivation of these formulae involved rather sophisticated assumptions and some intricate manipulation of the hypergeometric function and relied heavily on the solution of Schlömilch's integral equation. I have now found a much simpler formal solution by using Mehler's integral representation of the Legendre polynomial and the final formulae for the coefficients can be given in a more attractive form. As the results of my previous work have had several recent applications to physical problems, it seems worth while to give some details of this improved solution.

2. I start with the determination of the coefficients $A_{n}$ in the dual cosine series

$$
\begin{aligned}
& \sum_{n=0}^{\infty}(2 n+1) A_{n} \cos \left(n+\frac{1}{2}\right) x=f(x) \quad(0<x<c), \\
& \sum_{n=0}^{\infty} A_{n} \cos \left(n+\frac{1}{2}\right) x=0 \quad(c<x<\pi),
\end{aligned}
$$

and, by differentiation with respect to $x$, I write the second of these equations in the form

$$
\sum_{n=0}^{\infty}(2 n+1) A_{n} \sin \left(n+\frac{1}{2}\right) x=0 \quad(c<x<\pi)
$$

Using Mehler's integrals [2, p. 52],

$$
P_{n}(\cos \theta)=\frac{\sqrt{ } 2}{\pi} \int_{0}^{\theta} \frac{\cos \left(n+\frac{1}{2}\right) x d x}{(\cos x-\cos \theta)^{\frac{1}{2}}}=\frac{\sqrt{ } 2}{\pi} \int_{\theta}^{\pi} \frac{\sin \left(n+\frac{1}{2}\right) x d x}{(\cos \theta-\cos x)^{\frac{1}{2}}},
$$

equations (1) and (3) give

$$
\sum_{n=0}^{\infty}(2 n+1) A_{n} P_{n}(\cos \theta)=\left\{\begin{array}{cc}
F(\theta) & (0<\theta<c) \\
0 & (c<\theta<\pi)
\end{array}\right.
$$

where

$$
F(\theta)=\frac{\sqrt{ } 2}{\pi} \int_{0}^{\theta} \frac{f(x) d x}{(\cos x-\cos \theta)^{\frac{1}{2}}} .
$$

The coefficients in the Legendre series (5) are now given in the usual way by

$$
A_{n}=\frac{1}{2} \int_{0}^{c} F(\theta) P_{n}(\cos \theta) \sin \theta d \theta
$$


and the complete determination of the coefficients in the dual series (1) and (2) is provided by equations (7) and (6).

3. Formulae for the coefficients in the dual series

$$
\begin{aligned}
& \sum_{n=0}^{\infty} \frac{A_{n}}{2 n+1} \cos \left(n+\frac{1}{2}\right) x=f(x) \quad(0<x<c), \\
& \sum_{n=0}^{\infty} A_{n} \cos \left(n+\frac{1}{2}\right) x=0 \quad(c<x<\pi)
\end{aligned}
$$

are not quite so simple to find. Here, differentiation of (8) with respect to $x$ gives

$$
\sum_{n=0}^{\infty} A_{n} \sin \left(n+\frac{1}{2}\right) x=-2 f^{\prime}(x) \quad(0<x<c)
$$

and Mehler's integrals cannot be directly applied to equations (10) and (9) to give a Legendre series over the range $(0, \pi)$.

However, if equations (10) and (9) are multiplied by $2 \sin x$, they become

$$
\left.\begin{array}{l}
\sum_{n=0}^{\infty} A_{n}\left\{\cos \left(n-\frac{1}{2}\right) x-\cos \left(n+\frac{3}{2}\right) x\right\}=-4 f^{\prime}(x) \sin x \quad(0<x<c), \\
\sum_{n=0}^{\infty} A_{n}\left\{\sin \left(n-\frac{1}{2}\right) x-\sin \left(n+\frac{3}{2}\right) x\right\}=0 \quad(c<x<\pi),
\end{array}\right\}
$$

and these can be written in the form

$$
\left.\begin{array}{lr}
\left(A_{1}-A_{0}\right) \cos \frac{1}{2} x+\sum_{r=1}^{\infty}\left(A_{r+1}-A_{r-1}\right) \cos \left(r+\frac{1}{2}\right) x=-4 f^{\prime}(x) \sin x-2 A_{0} \cos \frac{1}{2} x \\
\left(A_{1}-A_{0}\right) \sin \frac{1}{2} x+\sum_{r=1}^{\infty}\left(A_{r+1}-A_{r-1}\right) \sin \left(r+\frac{1}{2}\right) x=0 \quad(c<x<\pi) . & (0<x<),
\end{array}\right\}
$$

Mehler's integrals (4) can now be applied to equations (12) to give with

$$
\left(A_{1}-A_{0}\right) P_{0}(\cos \theta)+\sum_{r=1}^{\infty}\left(A_{r+1}-A_{r-1}\right) P_{r}(\cos \theta)=\left\{\begin{array}{cc}
-2 F(\theta)-2 A_{0} & (0<\theta<c), \\
0 & (c<\theta<\pi),
\end{array}\right.
$$

$$
F(\theta)=\frac{2 \sqrt{ } 2}{\pi} \int_{0}^{\theta} \frac{f^{\prime}(x) \sin x d x}{(\cos x-\cos \theta)^{\frac{1}{2}}}
$$

and the coefficients in the Legendre series (13) are given by

$$
A_{1}-A_{0}=-\int_{0}^{c}\left\{F(\theta)+A_{0}\right\} P_{0}(\cos \theta) \sin \theta d \theta
$$




$$
A_{r+1}-A_{r-1}=-(2 r+1) \int_{0}^{c}\left\{F(\theta)+A_{0}\right\} P_{r}(\cos \theta) \sin \theta d \theta \quad(r=1,2,3, \ldots) .
$$

Now [2, p. 53]

$$
P_{n}^{\prime}(\cos \theta)=(2 n-1) P_{n-1}(\cos \theta)+(2 n-5) P_{n-3}(\cos \theta)+(2 n-9) P_{n-5}(\cos \theta)+\ldots
$$

the last term on the right-hand side being $3 P_{1}(\cos \theta)$ or $P_{0}(\cos \theta)$ according as $n$ is even or odd. Addition of equation (15) and equations (16) with $r=2,4,6, \ldots, n-1$ ( $n$ odd) or addition of equations (16) with $r=1,3,5, \ldots, n-1$ ( $n$ even) and use of (17) leads to

$$
\begin{aligned}
A_{n}-A_{0} & =-\int_{0}^{c}\left\{F(\theta)+A_{0}\right\} P_{n}^{\prime}(\cos \theta) \sin \theta d \theta \quad(n=1,2,3, \ldots) \\
& =-\int_{0}^{c} F(\theta) P_{n}^{\prime}(\cos \theta) \sin \theta d \theta-A_{0}\left[-P_{n}(\cos \theta)\right]_{0}^{c} .
\end{aligned}
$$

Hence

$$
A_{n}=A_{0} P_{n}(\cos c)-\int_{0}^{c} F(\theta) P_{n}^{\prime}(\cos \theta) \sin \theta d \theta \quad(n=1,2,3, \ldots)
$$

and equations (18) and (14) give $A_{n}$ in terms of $A_{0}$. The remaining coefficient $A_{0}$ can then be found by substitution in equation (8).

4. I next determine the coefficients $A_{n}$ in the dual sine series

$$
\begin{array}{ll}
\sum_{n=1}^{\infty} n A_{n} \sin n x=f(x) & (0<x<c), \\
\sum_{n=1}^{\infty} A_{n} \sin n x=0 & (c<x<\pi),
\end{array}
$$

and start by differentiating (20) with respect to $x$, obtaining

$$
\sum_{n=1}^{\infty} n A_{n} \cos n x=0 \quad(c<x<\pi) .
$$

Multiplication of (19) and (21) by $2 \sin \frac{1}{2} x$ and a slight rearrangement gives

$$
\left.\begin{array}{l}
\sum_{r=0}^{\infty}\left\{(r+1) A_{r+1}-r A_{r}\right\} \cos \left(r+\frac{1}{2}\right) x=2 f(x) \sin \frac{1}{2} x \quad(0<x<c), \\
\sum_{r=0}^{\infty}\left\{(r+1) A_{r+1}-r A_{r}\right\} \sin \left(r+\frac{1}{2}\right) x=0 \quad(c<x<\pi),
\end{array}\right\}
$$

and use of Mehler's integrals leads to

$$
\sum_{r=0}^{\infty}\left\{(r+1) A_{r+1}-r A_{r}\right\} P_{r}(\cos \theta)=\left\{\begin{array}{cc}
2 F(\theta) & (0<\theta<c), \\
0 & (c<\theta<\pi)
\end{array}\right.
$$


where in this case

$$
F(\theta)=\frac{\sqrt{2}}{\pi} \int_{0}^{\theta} \frac{f(x) \sin \frac{1}{2} x d x}{(\cos x-\cos \theta)^{\frac{1}{4}}}
$$

The coefficients in (22) are given by

$$
(r+1) A_{r+1}-r A_{r}=(2 r+1) \int_{0}^{c} F(\theta) P_{r}(\cos \theta) \sin \theta d \theta
$$

so that, summing for $r=0,1,2, \ldots, n-1$ and using (17),

$$
n A_{n}=\int_{0}^{c} F(\theta)\left\{P_{n}^{\prime}(\cos \theta)+P_{n-1}^{\prime}(\cos \theta)\right\} \sin \theta d \theta \quad(n=1,2,3, \ldots) .
$$

Equations (25) and (23) provide the complete solution in this case.

5. The coefficients in the dual series

$$
\begin{array}{ll}
\sum_{n=1}^{\infty} \frac{A_{n}}{n} \sin n x=f(x) & (0<x<c), \\
\sum_{n=1}^{\infty} A_{n} \sin n x=0 & (c<x<\pi)
\end{array}
$$

can be found in a similar way. Differentiation of (26) and multiplication of the result and of equation (27) by $2 \cos \frac{1}{2} x$ leads to

$$
\begin{aligned}
& A_{1} \cos \frac{1}{2} x+\sum_{r=1}^{\infty}\left(A_{r+1}+A_{r}\right) \cos \left(r+\frac{1}{2}\right) x=2 f^{\prime}(x) \cos \frac{1}{2} x \quad(0<x<c), \\
& A_{1} \sin \frac{1}{2} x+\sum_{r=1}^{\infty}\left(A_{r+1}+A_{r}\right) \sin \left(r+\frac{1}{2}\right) x=0 \quad(c<x<\pi),
\end{aligned}
$$

and use of (4) gives

$$
A_{1} P_{0}(\cos \theta)+\sum_{r=1}^{\infty}\left(A_{r+1}+A_{r}\right) P_{r}(\cos \theta)=\left\{\begin{array}{cc}
2 F(\theta) & (0<\theta<c), \\
0 & (c<\theta<\pi),
\end{array}\right.
$$

where

$$
F(\theta)=\frac{\sqrt{2}}{\pi} \int_{0}^{\theta} \frac{f^{\prime}(x) \cos \frac{1}{2} x d x}{(\cos x-\cos \theta)^{\frac{1}{2}}}
$$

The coefficients in (28) are given by

$$
\begin{gathered}
A_{1}=\int_{0}^{c} F(\theta) P_{0}(\cos \theta) \sin \theta d \theta \\
A_{r+1}+A_{r}=(2 r+1) \int_{0}^{c} F(\theta) P_{r}(\cos \theta) \sin \theta d \theta \quad(r=1,2,3, \ldots) .
\end{gathered}
$$


Alternate addition and subtraction of these equations and use of (17) yields

$$
A_{n}=\int_{0}^{c} F(\theta)\left\{P_{n}^{\prime}(\cos \theta)-P_{n-1}^{\prime}(\cos \theta)\right\} \sin \theta d \theta \quad(n=1,2,3, \ldots)
$$

and the complete solution of this case is given by equations (32) and (29).

\section{REFERENCES}

1. C. J. Tranter, Dual trigonometrical series, Proc. Glasgow Math. Assoc. 4 (1959), $49-57$.

2. W. Magnus and F. Oberhettinger (translated by J. Wermer), Special functions of mathematical physics (New York, 1949).

Royal Military College of Science

SHRIVENHAM 